\title{
Method of the Year 2014
}

\author{
Light-sheet fluorescence microscopy can image living samples in three dimensions with \\ relatively low phototoxicity and at high speed.
}

Just about everyone who has examined fluorescent samples under the microscope is aware of the constant struggle to have enough signal to see the labeled structures while also avoiding fluorophore bleaching. What may be less apparent, at least to those who image bright, robust or fixed samples, is how stressful and potentially toxic to living cells and tissues it is to illuminate them with high-intensity light. We have discussed this issue in a previous editorial (Nat. Methods 10, 1135, 2013).

Light-sheet fluorescence microscopy enables relatively gentle imaging of biological samples with high resolution in three dimensions (3D) and over long periods of time. Especially when combined with highspeed cameras, it is fast enough to capture cellular or subcellular dynamics. For its potential for fast, relatively gentle, volumetric imaging of biological samples, we have chosen light-sheet fluorescence microscopy as Method of the Year 2014.

The fundamental idea behind the technique is beautifully simple. Instead of illuminating or scanning the whole sample through the imaging objective, as in wide-field or confocal microscopy, one illuminates the sample from the side with a thin (practically 2D) plane or sheet of light. The emitted fluorescence is then detected from above or below the sample, along an axis perpendicular to the light sheet. Thus, optical sectioning-the ability to resolve structures in the $z$ plane-comes not from collecting photons solely from the focal point, as in confocal imaging, but from exciting only one plane at a time in the first place. In other words, light-sheet microscopy excites only the molecules at or near the focal plane, yielding lower phototoxicity and an improved ability to image living samples over time.

Based on a century-old technology first used to image colloids, this idea had been used in biological imaging during the twentieth century. But it was its application to living, fluorescently labeled zebrafish embryos by Ernst Stelzer and his group that began its recent biological renaissance. In a Commentary (p23), Stelzer discusses the origins, principles and power of this technique.

The rise of biological light-sheet microscopy also rests on the development of genetically encoded fluorescent proteins and the availability of labeled transgenic organisms. As described in a News Feature (p19), the physicists who initially developed modern light-sheet microscopes at the European Molecular Biology Laboratory,
Heidelberg, were surrounded by developmental biology laboratories generating and studying such fluorescent transgenics. The light-sheet renaissance thus required collaboration between the disciplines.

For the technology to be used to its fullest, this collaboration must continue. It is not trivial for biologists to implement light-sheet microscopy, as discussed in a Commentary by Pavel Tomancak and colleagues (p30); it requires creativity and interdisciplinary research teams. But with the release of commercial instruments, and efforts by the academic light-sheet development community to make the technology more accessible to users, imaging with light sheets is poised to give us new vistas into biological systems in ways we may not have thought possible.

Indeed, light-sheet fluorescence microscopy has recently been used to image living hearts and functioning brains and to track moving cells within developing embryos. It has been extended to multiview configurations and has been combined with super-resolution imaging, two-photon excitation and structured illumination. It can be used to powerful effect for fast 3D imaging in living cells and can be applied to optically cleared and fixed samples to produce spectacular static images of fluorescent structures.

The application of this technology in neuroscience is particularly exciting. As discussed in a Commentary by Misha Ahrens and colleagues (p27), the ability to image many cells simultaneously within the brain opens up the tantalizing possibility that some global properties of this most complex and mysterious of organs may be, at least to a first approximation, within reach.

Of course, none of this is easy. The volumes of data generated by light-sheet experiments, as discussed by all commentators in this issue, are daunting. New paradigms must be found for both data handling and analysis. Sample preparation for light-sheet imaging also departs entirely from the well-established methods known, though perhaps not loved, by biologists.

And, notably, the best light-sheet imaging is still of relatively small and transparent samples. Finding ways around scattering and aberrations in larger and more opaque samples continues to be important. Finally, users must still monitor potential phototoxicity, which is reduced but not necessarily absent with this technique. We predict an exciting few years as light sheets begin to shine through more biological research labs.

We wish our readers and contributors a happy 2015 ! 